Adapting to bilateral cochlear implants: Early post-operative device use by children receiving sequential or simultaneous implants at or before 3.5 years.

Karyn Louise Galvin and Kathryn Clare Hughes

Audiology, Hearing and Speech Sciences, Department of Otolaryngology, The University of Melbourne, Australia

\title{
Conflict declaration
}

The authors declare that they have no conflict-of-interest with regard to this manuscript or the conduct of the study reported. Financial support for this work was provided by the National Health and Medical Research Council (Project Grant No. 454318) and Audiology, Hearing and Speech Science, Department of Otolaryngology, The University of Melbourne.

Published in Cochlear Implants International, 2012, 13(2), 105-112

www.maneypublishing.com

www.maneypublishing.com/index.php/journals/cim/ 


\begin{abstract}
Objective: To classify adaptation difficulties, or lack thereof, experienced by a clinical population of young bilateral cochlear implant recipients.

Method: 46 of the first 48 children sequentially or simultaneously implanted at $\leq 3.5$ years at the Melbourne Clinic participated. Classification into categories was based on daily use of both implants at two months post-switch-on, with follow-up information obtained at 12 months. Results: The 37 Category 1 children wore both implants full-time at 2 months, and 35 still did so at 12 months. The two Category 2 children used both implants 4 hours daily at 2 months, but achieved full-time use within 12 months. The five Category 3 children used both implants for $\leq 1$ hour, with only three achieving full-time use within 12 months. The two Category 4 children did not use two implants at 2 months, and one still did not wear both implants at 12 months. There were weak/modest but significant relationships between category and each of time between implants and age at bilateral implantation.

Discussion: $95 \%$ of simultaneously and $70 \%$ of sequentially implanted children demonstrated full-time use within two months, and nearly all continued to do so at 12 months. Full-time use maximizes opportunities to develop listening skills. Monitoring device use is necessary for all children, especially when significant change occurs. For those experiencing difficulty in adapting, bilateral implant use usually increased over 12 months. Pre-operative counselling must include discussion of possible adaptation difficulties and raise the potential negative influence of age at bilateral implantation and time between implants.
\end{abstract}

Keywords: Bilateral cochlear implants; Children; Clinical practice; Pre-operative counselling 


\section{Introduction}

Cochlear implants have been provided to children with profound hearing loss since 1985. For many years, the standard care for such children was a unilateral cochlear implant. By the start of this century, over 20000 children worldwide had received a unilateral cochlear implant, with clear evidence of benefit for the majority of pediatric recipients (see, for example, Archbold et al., 2009a; Dowell et al., 2002; Geers et al., 2008; Sarant et al., 2001). More recently, a growing number of studies have demonstrated the potential for additional benefit to be gained from bilateral cochlear implants as compared with a single implant (see, for example, Dowell et al., in press; Galvin et al., 2010; Grieco-Calub et al., 2010; Lovett et al., 2010; Van Deun et al., 2010). As a result, bilateral implantation has become increasingly common worldwide: Peters et al. (2010) reported that the implant manufacturers' databases indicated there were 4986 children with bilateral implants. At many clinics, there are now three implant options: unilateral implantation, simultaneous bilateral implantation, or sequential bilateral implantation, so that preoperative counselling needs have become more complex and varied (Galvin et al., 2009b; Johnston et al., 2008). The importance of pre-operative counselling in reducing stress and helping parents to make an informed decision has been made clear in a number of studies (Johnston et al., 2009; Johnston et al., 2008; Most et al., 2001). This counselling should include discussion of the process of adapting to the new implant(s). Such a discussion is important not just in decision-making, but also in helping the parents to prepare for the early post-operative period. The establishment of appropriate expectations with regard to the implant process overall has been recognized as an important part of the pre-operative process (Zaidman-Zait et al., 2005).

Despite the number of children who have received cochlear implants, clinicians must still rely primarily on their own clinical experience and the anecdotal reports of other clinicians in 
providing information to families regarding the process of adapting to a new unilateral implant. There is limited information in the published literature regarding aspects of adaptation such as the time typically taken to achieve full-time device use and the ease with which this occurs, or the proportion of children who initially resist wearing their unilateral implant. Published articles reporting information on device use primarily focus on those children who are limited or nonusers of their implant in the longer-term (Archbold et al., 2009b; Ray et al., 2006; Watson et al., 2005). Watson and Gregory (2005) conducted parent and/or child interviews 5 to 7 years postimplantation, but did collect some information related to the early experiences of the five children involved, who were all non-users of their implant. Interestingly, it was reported that there "was resistance to the implant on the part of the children in each case from an early stage.....prior to the surgery in three instances" (pg 46). The attitude and/or experiences of these children in the early post-operative period is likely to be relevant to their outcome at 5 to 7 years postimplantation.

The information in the literature concerning the process of adapting to bilateral cochlear implants is also limited. Galvin et al. (2008) tracked the progress of ten children who received a second implant before 4 years of age. The authors reported that nine children were happy to wear their bilateral implants and were doing so full-time within 3 months, but that the tenth child had rejected the second implant. Galvin et al. (2010) tracked the progress of nine adolescents and young adults with sequential bilateral implants, and again reported the majority of participants were happy to use the bilateral implants and were doing so full-time within two months of receiving the second implant. A later report involved an additional 21 children, for a total of 40 participants who received sequential bilateral implants between the ages of 13 months and 19 years (Galvin et al., 2009a). In this larger group there was a greater proportion of children who 
struggled to achieve and/or maintain full-time bilateral use, particularly in the age range 3.5 to 10 years. These reports indicate that the ease with which children adapt to bilateral implant use may vary with age. There have been no reports in the literature of adaptation to bilateral implant use for large groups of children in specific age groups, or for any simultaneously implanted children. Such information is required if pre-operative counselling is to be appropriately targeted to individual children. It is also possible that the reports referred to above do not provide a valid representation of adaptation to bilateral implant use amongst the wider clinical population. The children tracked in the reports of Galvin et al. $(2010 ; 2009 a ; 2008)$ were all participants in an ongoing research project. Families and their children who volunteer to participate in such longterm research, which requires time and effort to attend test sessions and complete monthly interviews, may not be representative of the wider clinical population. Anecdotal information from clinicians has indicated that the proportion of children in the clinical population who experience difficulty adapting to two implants may be greater than that suggested by the reported progress of participants in the ongoing research program.

Clinicians need accurate information regarding the adaptation difficulties experienced by specific groups of children receiving bilateral implants. The clinician is then best placed to provide the pre-operative counselling required for families to make an informed choice, and also the postoperative support which minimizes the chance of adaptation problems occurring and helps to support the family in dealing with problems when they do occur. The aim of this study was to classify the adaptation difficulties, or lack of difficulty, experienced by a large group of children who were representative of the clinical population of children receiving sequential or simultaneous bilateral implants at or before 3.5 years of age at the Melbourne Implant Clinic. 


\section{Method}

The first 48 children to receive bilateral cochlear implants at or before 3.5 years of age at The Eye and Ear Hospital/The University of Melbourne Cochlear Implant Clinic were considered for this study. Two children were not included: one child who subsequently moved overseas and another child who was sequentially bilaterally implanted in an ear which had been previously implanted and then explanted. Outcomes are therefore reported here for 46 children who are numbered in order of their operation date for bilateral implantation. Note that very early postoperative information for eight of the children was previously reported in Galvin et al. (2008). Presented in Table 1 is age at implant and time between implant information for the overall group, as well as for both the 27 sequentially implanted children and the 19 simultaneously implanted children. Mean age at first implant was similar across the sequential and simultaneous groups, though mean age at bilateral implantation was later for the sequentially implanted children. All of the sequentially implanted children were consistent full-time users of their first implant, as this was a prerequisite for a second cochlear implant.

For 21 of the children, information regarding their adaptation to and use of their bilateral implants was obtained retrospectively from their hospital file and from the implant clinician who was the family's case-manager at the time of the fitting of the second (or both) implants. Approval for this data collection was obtained from the Human Research Ethics Committee of the Royal Victorian Eye and Ear Hospital (Project No. 04/564H/09). The remaining 25 children had also participated in a wider bilateral implant research project which included the prospective collection of data regarding adaptation to and use of the bilateral implants in monthly interviews with the primary caregiver. For these 25 children, additional information was obtained from their 
hospital file or implant clinician if required. Approval for this data collection was obtained from the Royal Victorian Eye and Ear Hospital (Project No. 02/506H/07).

Table 1: Mean (M), standard deviation (SD), and range of age at first implant, age at bilateral implantation, and time between implants for the sequentially implanted children, the simultaneously implanted children, and the overall participant group.

\begin{tabular}{|c|c|c|c|c|c|}
\hline \multirow{2}{*}{$\begin{array}{c}\text { Type of bilateral } \\
\text { implantation }\end{array}$} & \multicolumn{2}{|c|}{$\begin{array}{l}\text { Age at first implant } \\
\text { (yr;mo) }\end{array}$} & $\begin{array}{l}\text { Age at bilateral } \\
\text { implantation (yr;mo) }\end{array}$ & \multicolumn{2}{|c|}{$\begin{array}{c}\text { Time b/w implants } \\
\text { (yr;mo) }\end{array}$} \\
\hline & $\mathrm{M}(\mathrm{SD})$ & Range & $M(S D)$ & $\mathrm{M}(\mathrm{SD})$ & Range \\
\hline Any type $(n=46)$ & $1 ; 6(0 ; 7)$ & $0 ; 7-3 ; 6$ & $0 ; 9-3 ; 6$ & $0 ; 7(0 ; 8)$ & $0 ; 0-2 ; 1$ \\
\hline Sequential $(n=27)$ & $1 ; 4(0 ; 6)$ & $0 ; 7-2 ; 3$ & $2 ; 3(0 ; 8) \quad 1 ; 1-3 ; 6$ & $0 ; 11(0 ; 7)$ & $0 ; 3-2 ; 1$ \\
\hline Simultaneous $(n=19)$ & $1 ; 8(0 ; 8)$ & $0 ; 9-3 ; 6$ & $1 ; 8(0 ; 8)$ & NA & NA \\
\hline
\end{tabular}

The children were classified into one of four categories based on the amount of time the two implants were used together at the two-month post switch-on point. This two-month point was selected so that minor issues around consistent device use in the early weeks, such as those which may occur before an appropriate map is established, were not included as "adaptation difficulties". Children who were cooperative in wearing both implants full-time at the two-month point were classified into Category 1. "Full-time" was defined as at least $90 \%$ of waking hours, so that behaviours such as not wearing both implants between bath and bedtime were not considered "adaptation difficulties". Children who wore the two implants together for a number of hours but not full-time at the two-month point were classified into Category 2. Children who 
wore the two implants together but only for a very limited amount of time were classified into Category 3, and children who did not wear the two implants together at all were classified into Category 4. For children classified into Categories 2 to 4, information regarding bilateral implant use was obtained up to the 12-month post switch-on point. For the children classified into Category 1, confirmation that full-time use of both implants was still occurring at the 12-month point was obtained.

\section{Results}

At the two-month post-operative point, 37 of the 46 children were classified into Category 1. This group included $95 \%$ of simultaneously implanted children and $70 \%$ of sequentially implanted children. When followed up at the 12-month post switch-on point, 35 of these Category 1 children were using their two implants full-time. The remaining two children, both of whom were simultaneously implanted, had rejected their implants. One of these children had autism spectrum disorder, and the other child was being assessed for a neurological disorder.

The remaining 9 children were classified into Category $2(n=2)$, Category $3(n=5)$, or Category $4(\mathrm{n}=2)$. Only one of these children was simultaneously implanted, and this child was classified into Category 3. Figures 1, 2 and 3 present the typical hours of bilateral implant use per day for the children in each of Category 2, 3 and 4 respectively in the first 12 months after switch-on of the second or both implants. As shown in Figure 1, the two children in Category 2 were only using their two implants together for around 4 hours per day at the two-month point. These children had a preference for one implant over the other, which they indicated verbally or behaviourally. There was a tendency for the children to use only the preferred implant when 
tired, unwell, or upset. Rates of bilateral implant use improved for these children over the ensuing 12 months, with both using the two implants full-time by the 10-month point.

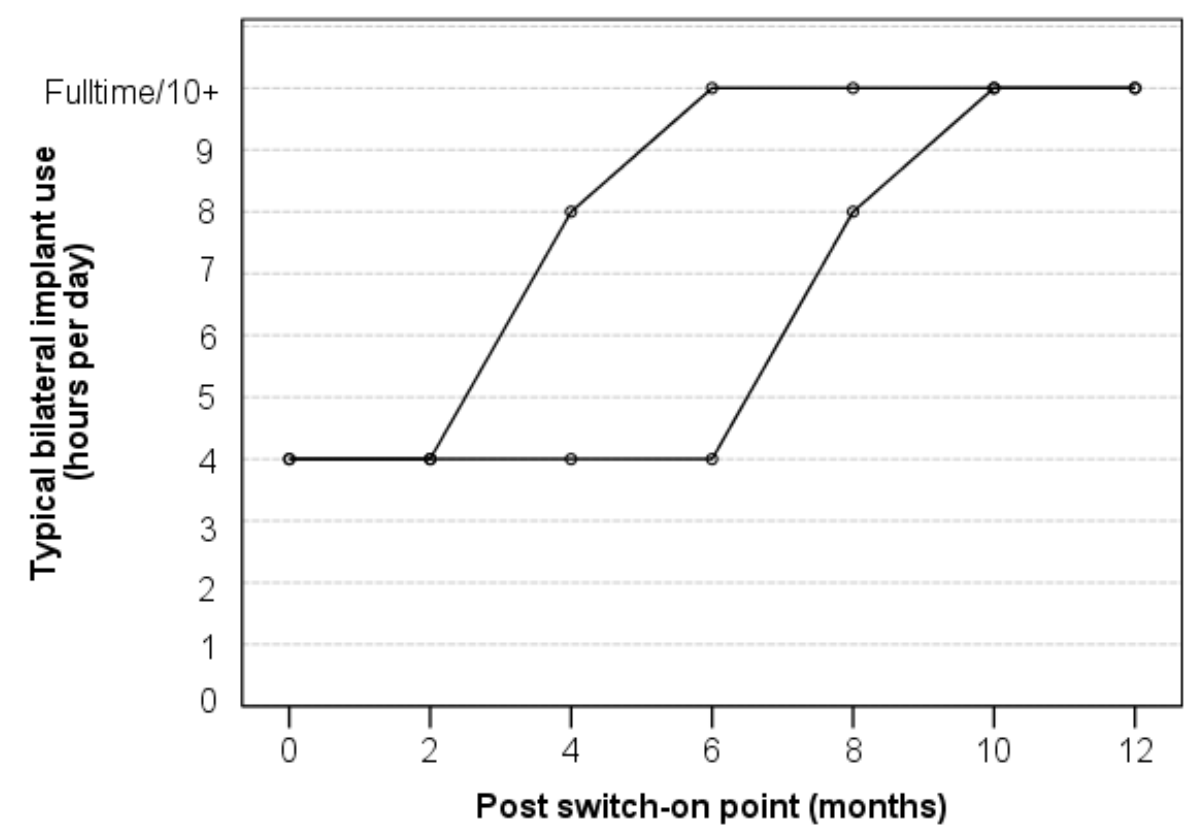

Figure 1: Typical hours of bilateral implant use per day in the first 12 months post switch-on for the two children in Category 2.

As shown in Figure 2, bilateral usage at the two-month point was a maximum of one hour per day for the five children in Category 3. The four sequentially implanted children in this group had a clear preference for their first implant. The simultaneously implanted child also had a clear preference for one implant over the other. Younger children in this group removed the coil of the non-preferred implant many times per day, and older children required encouragement from caregivers if they were to put on the non-preferred implant, and ongoing encouragement if they were to keep it on. Rates of bilateral implant use improved for these children over the 12 months, although improvement was sometimes slow and/or not maintained. By the 10-month point three of these five children were using the two implants full-time. 


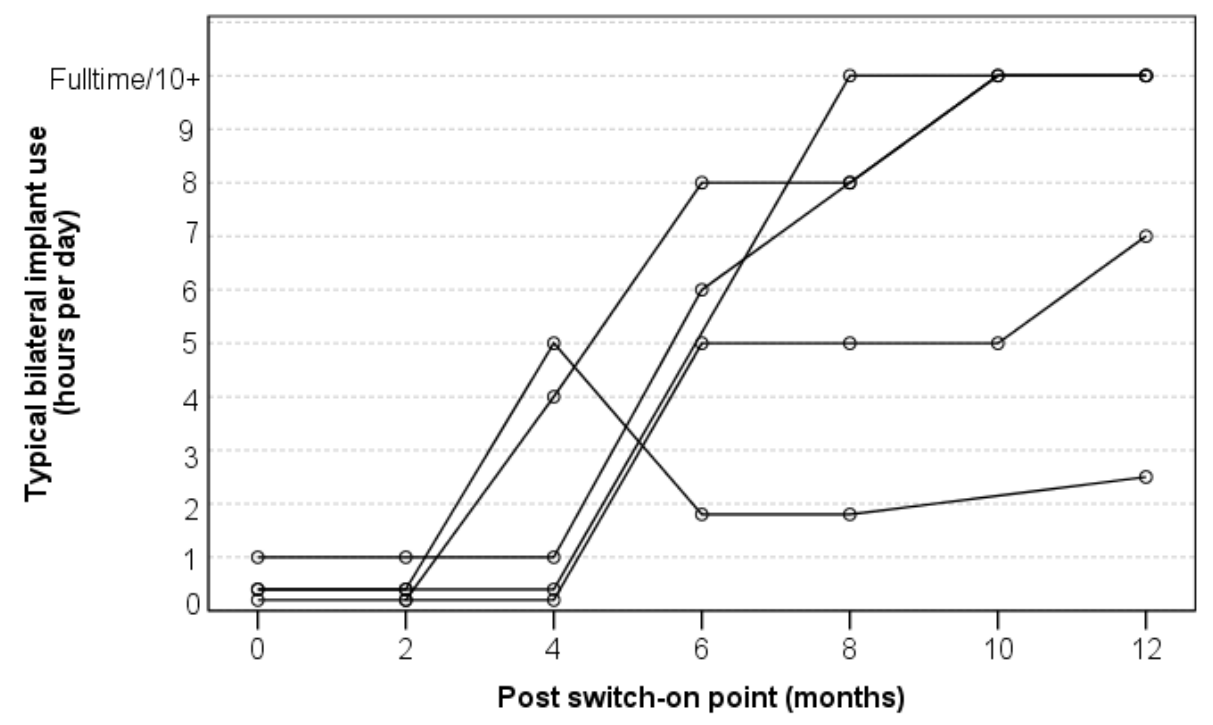

Figure 2: Typical hours of bilateral implant use per day in the first 12 months post switch-on for the five children in Category 3.

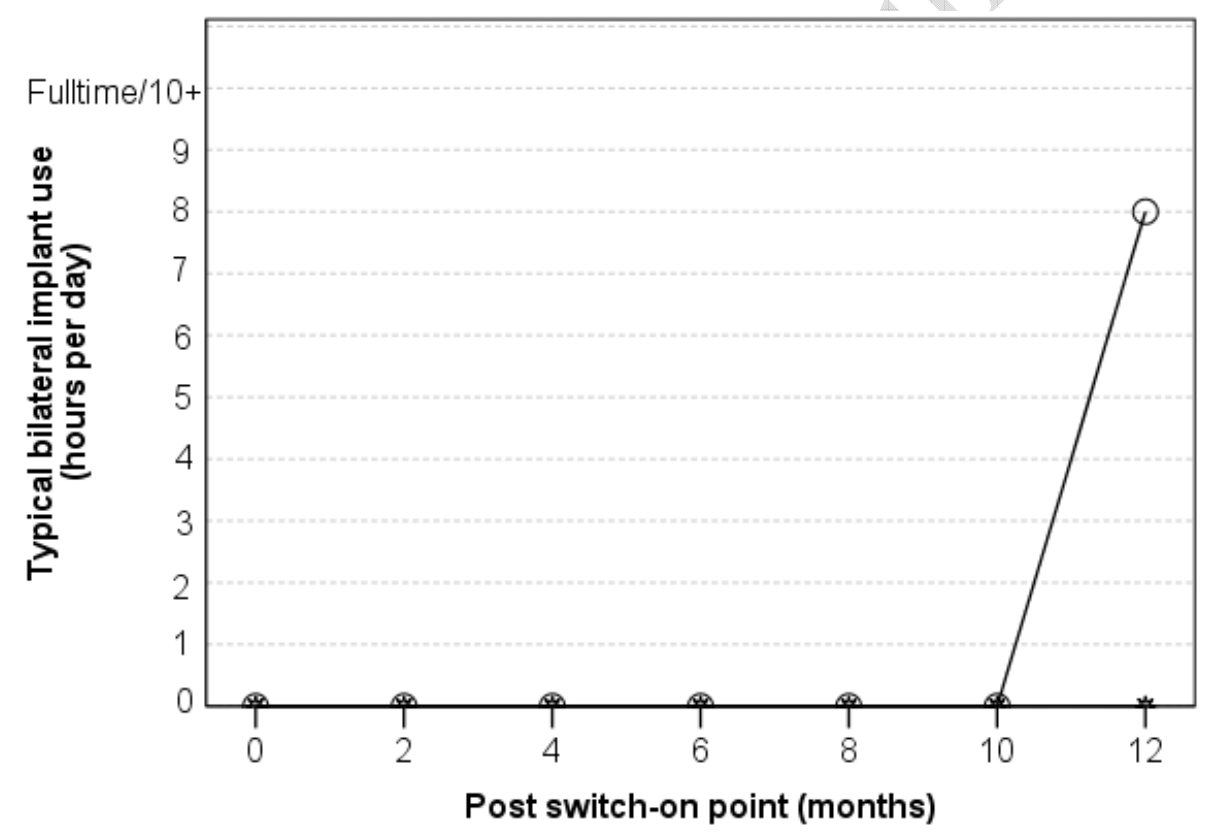

Figure 3: Typical hours of bilateral implant use per day in the first 12 months post switch-on for the two children in Category 4.

As shown in Figure 3, the two children in Category 4 had no regular use of the two implants together at the two-month point. For one of these children, usage increased significantly to 8 
hours per day by the 12-month point. The other participant was still not wearing the second implant at the 12-month point.

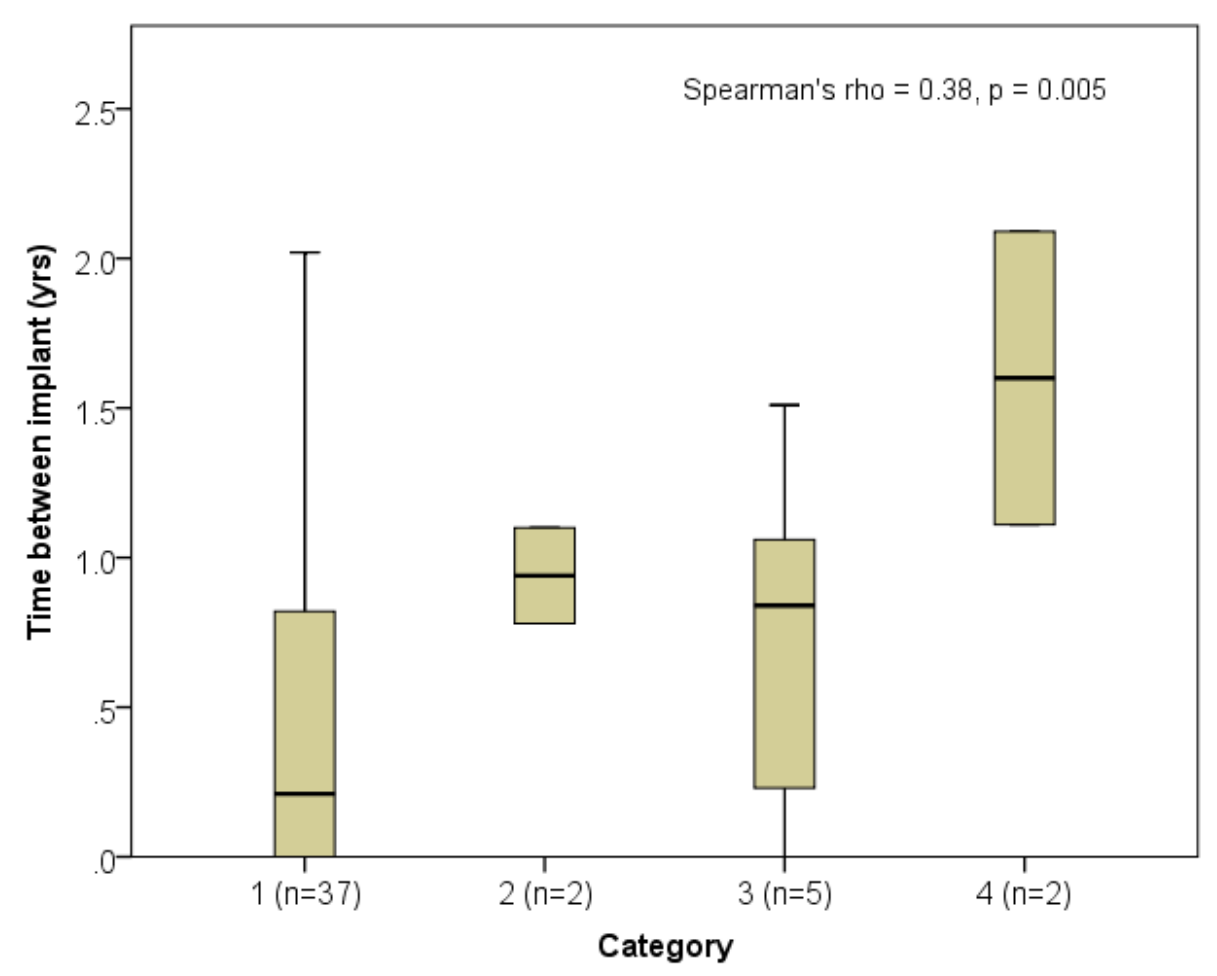

Figure 4: Time between implants as a function of the category into which children were classified based on their hours of daily use of their bilateral implants at the 2-month post switchon point. The solid bar indicates the median, the box represents the inter-quartile range, and the ends of the whiskers indicate the minimum and maximum values.

Figures 4 and 5 present box plots of the time between implants and the age at bilateral implantation respectively for each of the four categories. In both figures there is clear overlap in the range for most categories. It is important to note the small sample sizes in categories 2 to 4 . One-tailed Spearman's Rank Order correlation coefficients indicated a weak to modest, but nevertheless statistically-significant, relationship between category and the factors of time between implants $(\rho=0.38, p=0.005)$ and age at bilateral implantation $(\rho=0.27, p=0.033)$. Calculation of the Spearman coefficients for the 27 children with sequential implants only, 
indicated no significant relationship between category and the factors of time between implants ( $\rho=0.28, p=0.079)$ or age at bilateral implantation $(\rho=0.29, p=0.071)$ for this subgroup.

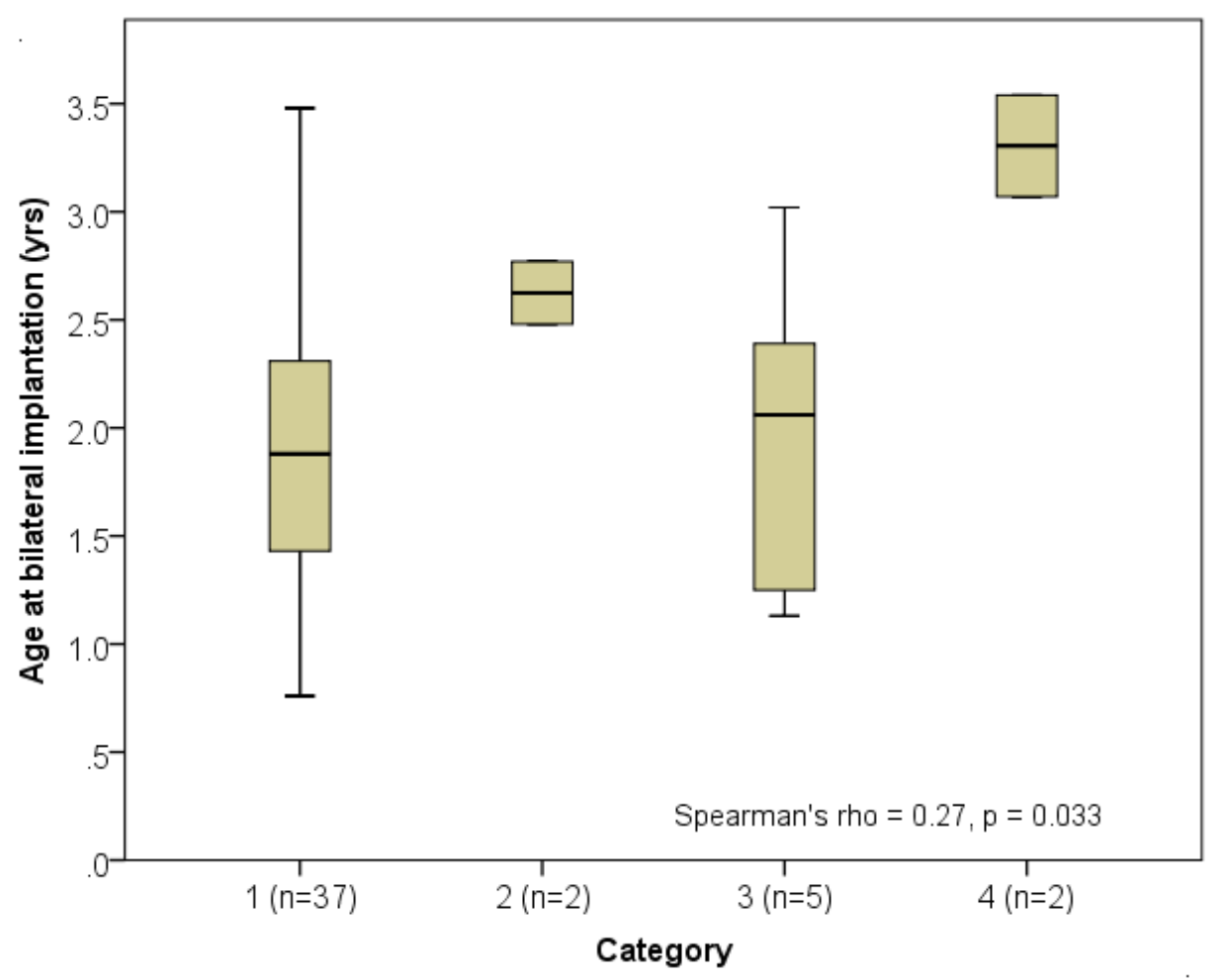

Figure 5: Age at bilateral implantation as a function of the category into which children were classified based on their hours of daily use of their bilateral implants at the 2-month post switchon point. The solid bar indicates the median, the box represents the inter-quartile range, and the ends of the whiskers indicate the minimum and maximum values.

\section{Discussion}

Of the 46 children receiving bilateral cochlear implants at or before 3.5 years of age, $95 \%$ of simultaneously implanted children and $70 \%$ of sequentially implanted children were using their 
devices full-time within two months. Although full-time device use does not guarantee a particular level of benefit, it maximizes the child's opportunity to develop their listening skills with each implant and to develop binaural listening skills. At the time a child is implanted, the implant clinician cannot influence many factors which may contribute to the child's benefit from their implant(s), such as the age at onset and physiological consequences of the child's hearing loss, the level and quality of pre-operative hearing, pre-operative hearing aid use and chronological age. A primary area where the implant clinician can have an impact is in establishing and supporting full-time device use. The results of this study indicate that this is fairly straightforward to achieve for the majority of children implanted bilaterally at or before 3.5 years of age, particularly when the implants are received simultaneously.

The information obtained at the 12-month post-operative point indicated that nearly all children (95\%) who were wearing their implants full-time at the two-month point were also doing so at the 12-month point. This reinforces the importance of achieving good patterns of device use in the early post-operative period. The two children in Category 1 who were no longer full-time users at the 12-month point both had an additional disability. Given the serious impact of longterm device rejection on the development of listening, speech production, and oral language skills, the outcome for these two children emphasizes the importance of continued monitoring of device use amongst children. Such monitoring is important even for those children who have accepted their implants and have been using the devices full-time. Significant events in the child's life, such as illness or a dramatic change in circumstances, can trigger stress which may manifest itself as a negative attitude towards implant use. A negative attitude may also develop in response to hardware faults (particularly ongoing intermittent faults) or the need for a significant map change which affect the quality of sound received by the child. Any suggestion 
of inconsistent use of either device is an important marker for the parents and clinician. Bilaterally implanted children can express their stress or frustration by rejecting one implant whilst maintaining access to sound via the other implant. Parents and clinicians may consider such behaviour to be a relatively insignificant temporary problem because the child can continue to function relatively normally, however a significant break in device use then requires reintroduction of and adaptation to that implant. Intervening early when issues with device use first appear maximizes the chance that full-time device use can be maintained or quickly reestablished.

Despite the ease with which the majority of children adapted to bilateral implant use, adaptation was not easy for a significant proportion (almost 20\%) of the group. It was difficult to conclusively determine why adaptation was not easy for these individuals. Prior to the introduction of implant recognition technology, if the coil of the first implant was placed over the implant package on the second implanted side over stimulation was possible. Parent reports indicated that one child in Category 3 was resistant to using his second implant after this occurred. Another child in Category 4 developed a significant fear of the second implant and refused to be near it. This may have been due to an incident in hospital when the child placed the incorrect coil over the second implant package; the child may have been reacting over stimulation or to the fear expressed by the parents when this occurred. The long and unexpected absence of the mother from the family home may have also been a factor in the child's behaviour at this time. After 10 months work by parents and clinicians to reduce the anxiety associated with the second implant, this child began wearing the device. The remaining children simply did not want to wear the second implant. It can only be speculated as to whether this was due to a dislike of the sound provided via that implant, a dislike of change, or the child wishing to exert control. In 
some cases, different behavioural management techniques may have been successful in encouraging full-time use more quickly.

It is vital that the potential outcome of difficulty adapting to two implants, and the factors which may contribute to it, are discussed during pre-operative counseling so that parents are in an informed position. Strategies for managing bilateral implant use in the immediate post-operative period should also be discussed pre-operatively. This is particularly important if the parent feels their child may find change difficult. It is important that the caregivers are clear on what their own expectations are for implant use and how they plan to achieve that level of use. Assuming full-time bilateral use is the caregivers' goal for the child, this can be expected, encouraged and rewarded from the time of switch-on. This is arguably the best time for this to occur, as the initial map(s) is/are typically set conservatively in terms of the maximum amount of electrical stimulation provided. Maximum levels of electrical stimulation and so, theoretically, the level of sound perceived, are likely to be increased in subsequent mapping sessions. If consistent bilateral implant use has been established as a daily pattern of behavior early-on in this process, the child is given maximum opportunity to adapt gradually to the change in sound as the map is optimized over the first few weeks post-operatively. This may be particularly important for children receiving sequential bilateral implants as they have a first implant with which to compare the second implant, and potentially find it lacking in clarity or quality. Clinicians have an important role to play in supporting caregivers in establishing and maintaining bilateral implant use. Clinicians can also gain important information from caregivers' reports of device use which may influence the process of optimizing the map.

For the current group, when bilateral implants use was not full-time at the two-month point, some difficulties with consistent bilateral implant use persisted. Only five of the nine children in 
Categories 2 to 4 were using both implants full-time at the 12-month point. However, more positively, eight of the children did increase their daily use of their bilateral implants between the 2- and 12-month points, and only one child remained a non-user of their bilateral implants (i.e., their second implant) at the 12-month point. This result demonstrates that it is worthwhile for parents and clinicians to persist in encouraging and rewarding bilateral implant use even if the child has difficulty adapting to the two devices.

Numerous studies have suggested that receiving bilateral implants at a younger age and having a shorter time between implants may be positively associated with greater benefit being gained (Manrique et al., 2007; Peters et al., 2007; Scherf et al., 2007; Wolfe et al., 2007). It is difficult to isolate the individual influence of these two factors given that they are usually related, with only older children able to have a long period between implants, and younger children being far more likely than older children to be simultaneously implanted. In the present study, these factors of age at bilateral implantation and time between implants were also positively associated with adaptation category. Although the relationships were statistically significant, they were not strong and there was overlap across the categories in terms of the range of time between implants and age at second implant. This indicates that, although younger children with a shorter delay between implants are less likely to have difficulty adapting to bilateral implants, individuals who are older and/or have more time between implants are not necessarily going to have difficulty adapting. The important point to make is that clinicians need to include discussion of the potential influence of age at bilateral implantation and time between implants in pre-operative counselling. This is relevant both for parents considering whether to minimize the time between implants and the age at bilateral implantation by opting for simultaneous implants, and for parents deciding whether and when to obtain a second sequential bilateral implant. 
Interestingly, when the relationship between adaptation category and the factors of age at bilateral implantation and time between implants was examined for the sequentially implanted children only, no significant relationships were found. It may be that, for a child who is not simultaneously implanted and/or implanted at a very young age, time between implants and age at bilateral implantation may have less influence on adaptation than other factors. Such factors may be associated with the child (e.g., personality and flexibility) or the parents (e.g., expectations and behavior management strategies implemented). It is also possible that, with such small numbers of children in each of categories 2 to 4 , the analysis was not sufficiently powerful to detect a relationship.

The significant relationships evident for the overall group between adaptation category and the factors of age at bilateral implantation and time between implants were likely strongly influenced by the very high proportion of simultaneously implanted children who had no difficulty adapting to bilateral implant use. The two groups differed in that the simultaneous group had, by definition, zero months between implants (compared with a mean of $11 \mathrm{mths}$ for the sequential group) and were significantly younger at bilateral implantation (mean 1 yr 8 mths versus 2 yrs 3 mths; $\mathrm{t}(44)=3.15, \mathrm{p}=0.003)$. Although it is not possible to separate out the influence of these factors, the authors propose that having zero time between implants is likely to be the more important factor. The first reason for this is that the simultaneously implanted child will only have experience of the sound of the two implants together. In contrast, the sequentially implanted child has experience with a different sound percept (i.e., that from the first implant alone) with which to make a comparison, and this comparison may be a negative one. Secondly, for the simultaneously implanted child, the two implants are likely to provide a similar percept in terms of familiarity, quality, and information content. Such a child is therefore less likely to have 
a non-preferred implant that they are tempted to remove. Thirdly, the simultaneously implanted child may perceive the two implants as a "set"; if such a child does not like the sound percept at all they may remove both implants, however it may not occur to them to remove just one implant.

It is important to note a limitation of the present study which may be important for clinical practice. The present participant group was drawn from the first 48 children to receive bilateral implants at the Melbourne Implant Clinic. It is fair to say, regarding the earliest bilateral implant operations, that the families involved were entering relatively uncharted territory. These 46 children and their families may not be entirely representative of the clinical population currently receiving bilateral implants as the clinical situation is now different. For example, more children with additional disabilities are now being considered for a second implant, simultaneous implants are more routinely offered, and parents now have some evidence on which to base their decision and to form their expectations of the post-operative experience.

In considering areas for future research, it will be of interest to revisit the group of children in the present study to collect information regarding device use in the longer term. This information can then be related back to the data reported here on the children's early post-operative adaptation to bilateral implant use. Whether early difficulties adapting to bilateral implant use are predictive of long-term limited device use and/or limited benefit are important clinical questions. There are also particularly interesting individual cases, the longer-term outcomes of which will be of interest to clinicians managing children with bilateral implants. For example, one child in Category 4 was not using the second implant at all at 12-months; at the time of writing the child's school and clinicians were attempting to re-introduce the second implant even though the child was now more than 3 years post-operative. Also, only one simultaneously implanted child had difficulty adapting to bilateral implant use, and this child had a strong 
preference for one implant over the other. Although full-time bilateral use was later reported for this child, it will be of interest to follow-up to determine if this initial bias appears evident in long-term patterns of device use and/or perceptual performance. Another area for further research is the process of adapting to bilateral implants for older children. Clinical experience and the reports of Galvin et al. (2009a) suggest that older children have more difficulty adapting than younger children. It is vital that clinicians have age-appropriate evidence on which to base their pre-operative counselling.

\section{Conclusion}

In this group of children receiving bilateral cochlear implants at or before 3.5 years of age, by far the majority of children had no difficulty adapting to the use of two implants. Even when fulltime use is establish quickly, clinicians and parents need to monitor device use, especially around the time of significant events in the child's life. Of those children experiencing some difficulty adapting to bilateral implant use, the majority were sequentially implanted. The majority of these children increased their daily use of bilateral implants over the first 12 months post-operatively. There were significant, though only weak to modest, positive relationships between the category of difficulty adapting to bilateral implants and the time between implants as well as the age at bilateral implantation. These relationships did not appear to be present for the sequentially implanted group alone. It is vital that clinicians discuss the possibility of difficulties in the adaptation process with families considering bilateral implantation, and raise the potential influence of age at bilateral implantation and time between implants. 


\section{Acknowledgements}

The authors are very grateful to the families and to the staff of the Royal Victorian Eye and Ear Hospital/The University of Melbourne Cochlear Implant Clinic who provided information for this project. The authors also thank Meredith Prain, Colleen Holt, Alexandra Tomov, Mansze Mok, and Marie-Chantal Moll-Vignes for their contribution to data collection.

\section{References}

Archbold, S. and O'Donoghue, G. M. (2009a) 'Cochlear implantation in children: current status', Paediatrics and Child Health 19 (10): 457-463.

Archbold, S. M., Nikolopoulos, T. P. and Lloyd-Richmond, H. (2009b) 'Long-term use of cochlear implant systems in paediatric recipients and factors contributing to non-use', Cochlear Implants International 10 (1): 25-40.

Dowell, R., Galvin, K., Dettman, S., Hughes, K. C. and van Hoesel, R. (in press) 'Bilateral Cochlear Implants in Children', Seminars in Hearing.

Dowell, R. C., Dettman, S. J., Blamey, P. J., Barker, E. J. and Clark, G. M. (2002) 'Speech perception in children using cochlear implants: prediction of long-term outcomes', Cochlear Implants International 3 (1): 1-18.

Galvin, K. L., Hughes, K. C. and Mok, M. (2010) 'Can adolescents and young adults with prelingual hearing loss benefit from a second, sequential cochlear implant?', International Journal of Audiology 49 (5): 368-377. 
Galvin, K. L., Hughes, K. C., Mok, M., Holland, J., Fewster, L., Tomov, A., et al. (2009a) 'Documenting progress with sequential, bilateral cochlear implants: Reported device use and performance milestones achieved by toddlers, children and young adults', 9th European Symposium on Pediatric Cochlear Implantation. May 14-17 Warsaw, Poland.

Galvin, K. L., Leigh, J. R. and Hughes, K. C. (2009b) 'How we do it: Clinical management of the child receiving a second, bilateral cochlear implant', Cochlear Implants International 10 (2): 84-91.

Galvin, K. L., Mok, M., Dowell, R. C. and Briggs, R. J. (2008) 'Speech detection and localization results and clinical outcomes for children receiving sequential bilateral cochlear implants before four years of age', International Journal of Audiology 47 (10): 636-646.

Geers, A., Tobey, E., Moog, J. and Brenner, C. (2008) 'Long-term outcomes of cochlear implantation in the preschool years: From elementary grades to high school', International Journal of Audiology 47 (S2): S21-S30.

Grieco-Calub, T. M. and Litovsky, R. Y. (2010) 'Sound localization skills in children who use bilateral cochlear implants and in children with normal acoustic hearing', Ear \& Hearing 31 (5): 645-56.

Johnston, J. C., Durieux-Smith, A., Angus, D., O'Connor, A. and Fitzpatrick, E. M. (2009) 'Bilateral paediatric cochlear implants: A critical review', International Journal of Audiology 48 (9): 601-617. 
Johnston, J. C., Durieux-Smith, A., Fitzpatrick, E., O'Connor, A., Benzies, K. and Angus, D. (2008) 'An assessment of parents decision-making regarding paediatric cochlear implants', Canadian Journal of Speech-Language Pathology and Audiology 32 (4): 169-182.

Lovett, R. E., Kitterick, P. T., Hewitt, C. E. and Summerfield, A. Q. (2010) 'Bilateral or unilateral cochlear implantation for deaf children: an observational study', Archives of Disease in Childhood 95 (2): 107-12.

Manrique, M., Huarte, A., Valdivieso, A. and Perez, B. 2007. Bilateral sequential implantation in children. Audiological Medicine 5 (4): 224-231.

Most, T. and Zaidman-Zait, A. (2001) 'The needs of parents of children with cochlear implants', Volta Review 103 (2): 99-113.

Peters, B., Litovsky, R., Parkinson, A. and Lake, J. (2007) 'Importance of age and postimplantation experience on speech perception measures in children with sequential bilateral cochlear implants', Otology \& Neurotology 28 (5): 649-657.

Peters, B. R., Wyss, J. and Manrique, M. (2010) 'Worldwide trends in bilateral cochlear implantation', The Laryngoscope 120 (S2): S17-S44.

Ray, J., Wright, T., Fielden, C., Cooper, H., Donaldson, I. and Proops, D. W. (2006) 'Non-users and limited users of cochlear implants', Cochlear Implants International 7 (1): 49-58.

Sarant, J. Z., Blamey, P. J., Dowell, R. C., Clark, G. M. and Gibson, W. P. (2001) 'Variation in speech perception scores among children with cochlear implants', Ear \& Hearing. 22 (1): $18-28$ 
Scherf, F., van Deun, L., van Wieringen, A., Wouters, J., Desloovere, C., Dhooge, I., et al. (2007) 'Hearing benefits of second-side cochlear implantation in two groups of children', International Journal of Pediatric Otorhinolaryngology 71 (12): 1855-1863.

Van Deun, L., van Wieringen, A., Scherf, F., Deggouj, N., Desloovere, C., Offeciers, F. E., et al. (2010) 'Earlier intervention leads to better sound localization in children with bilateral cochlear implants', Audiology and Neurotology 15 (1): 7-17.

Watson, L. M. and Gregory, S. (2005) 'Non-use of cochlear implants in children: child and parent perspectives', Deafness \& Education International 7 (1): $43-58$.

Wolfe, J., Baker, S., Caraway, T., Kasulis, H., Mears, A., Smith, J., et al. (2007) '1-year postactivation results for sequentially implanted bilateral cochlear implant users', Otology \& Neurotology 28 (5): 589-96.

Zaidman-Zait, A. and Most, T. (2005) 'Cochlear Implants in Children with Hearing Loss: Maternal Expectations and Impact on the Family', Volta Review 105 (2): 129-150. 


\section{University Library}

\section{- M M N E R VA A gateway to Melbourne's research publications}

Minerva Access is the Institutional Repository of The University of Melbourne

Author/s:

Galvin, KL;Hughes, KC

Title:

Adapting to bilateral cochlear implants: early post-operative device use by children receiving sequential or simultaneous implants at or before 3.5 years.

Date:

2012-05

\section{Citation:}

Galvin, K. L. \& Hughes, K. C. (2012). Adapting to bilateral cochlear implants:

early post-operative device use by children receiving sequential or simultaneous implants at or before 3.5 years.. Cochlear Implants Int, 13 (2), pp.105-112. https:// doi.org/10.1179/1754762811Y.0000000001.

Persistent Link:

http://hdl.handle.net/11343/58701 